\title{
Chaotic renormalization-group trajectories
}

Damgaard, Poul H.; Thorleifsson, G.

Published in:

Physical Review A

Link to article, DOI:

10.1103/PhysRevA.44.2738

Publication date:

1991

Document Version

Publisher's PDF, also known as Version of record

Link back to DTU Orbit

\section{Citation (APA):}

Damgaard, P. H., \& Thorleifsson, G. (1991). Chaotic renormalization-group trajectories. Physical Review A, 44(4), 2738-2741. https://doi.org/10.1103/PhysRevA.44.2738

\section{General rights}

Copyright and moral rights for the publications made accessible in the public portal are retained by the authors and/or other copyright owners and it is a condition of accessing publications that users recognise and abide by the legal requirements associated with these rights.

- Users may download and print one copy of any publication from the public portal for the purpose of private study or research.

- You may not further distribute the material or use it for any profit-making activity or commercial gain

- You may freely distribute the URL identifying the publication in the public portal

If you believe that this document breaches copyright please contact us providing details, and we will remove access to the work immediately and investigate your claim. 


\title{
Chaotic renormalization-group trajectories
}

\author{
P. H. Damgaard \\ MIDIT, The Technical University of Denmark, DK-2800 Lyngby, Denmark \\ G. Thorleifsson \\ The Niels Bohr Institute, University of Copenhagen, DK-2100 Copenhagen, Denmark \\ (Received 19 November 1990)
}

\begin{abstract}
Under certain conditions, the renormalization-group flow of models in statistical mechanics can change dramatically under just very small changes of given external parameters. This can typically occur close to bifurcations of fixed points, close to the complete disappearance of fixed points, or in regions where the renormalization-group flow becomes chaotic. We present some explicit examples of these phenomena for the case of a Lie group valued spin-model analyzed by means of a variational realspace renormalization group. By directly computing the free energy of these models around the parameter regions in which such nontrivial modifications of the renormalization-group flow occur, we can extract the physical consequences of these phenomena.
\end{abstract}

When a given theory is analyzed by means of a renormalization-group ( $R G)$ technique, certain features, not easily disentangled by other methods, can become apparent. One typically wishes to focus on those aspects of the renormalization transformation that contain information about universal properties (critical exponents, certain amplitude ratios, etc.) of the class of theories one is studying. This normally restricts attention to the linearized neighborhood of critical fixed points (see, e.g., Ref. [1] for a review). But essentially all other thermodynamic functions of any given model can be extracted from the RG transformation for any range of couplings by computing the free energy and its derivatives. Thus also the RG flow far from critical fixed points can be related to physical observables. Still, it is also known that the actual RG flow can change drastically without affecting the physics.

A typical example illustrating the above remarks concerns the existence of redundant operators [2]. The critical point Hamiltonian may be perturbed in redundant directions without affecting any physical quantities. Such perturbations away from the fixed point Hamiltonian are simply unphysical. Thus although certain aspects of the RG flow directly give us physical information, other features are simply artifacts of the chosen RG transformation.

To make these general statements more concrete, we define a real-space $(\mathrm{RG})$ transformation $\mathcal{R}_{[b]}: H \rightarrow H^{\prime}$ bringing us from Hamiltonian $H$ to the renormalized Hamiltonian $H^{\prime}$ (here $b$ denotes the spatial rescaling factor). In the space of couplings $\{\mathbf{K}\}=\left\{K_{1}, K_{2}, \ldots\right\}$ we can (in a slightly imprecise notation) write this as the iterative equation

$$
\mathbf{K}^{\prime}=\mathscr{R}_{[b]}(\mathbf{K}) \text {. }
$$

In particular, for the singular part of the free energy per degree of freedom one obtains a recursive relation [1]

$$
f(\mathbf{K})=g(\mathbf{K})+b^{-d} f\left(\mathcal{R}_{[b]}(\mathbf{K})\right),
$$

where $d$ is the dimension of space. The function $g(\mathbf{K})$ is simply the constant term of the Hamiltonian. It will in general be an analytic function.

Provided that $f\left(R_{[b]}^{(N)}(\mathbf{K})\right)$ is sufficiently bounded as $N \rightarrow \infty$, the solution to Eq. (2) can be written as

$$
f(\mathbf{K})=\sum_{n=0}^{\infty} b^{-d n} g\left(\mathcal{R}_{[b]}^{(n)}(\mathbf{K})\right) .
$$

Already from these elementary considerations we are able to discern certain features arising from a modification of the RG flow, and see how this nevertheless can lead to "invariant" physical results. What is required is clearly, as can be seen from Eq. (3), that a change of flow [the behavior of $\mathcal{R}_{[b]}^{(n)}(\mathbf{K})$ ] is precisely compensated by a change in the nonsingular function $g(\mathbf{K})$ of the free energy. In this way one can achieve that a change in $\mathcal{R}_{[b]}(\mathbf{K})$ and $g(\mathbf{K})$ leads to the same physical free energy for all couplings $K_{i}$. This also makes it obvious that one should be very cautious about assuming that radically different $R G$ flows necessarily should correspond to different thermodynamic behavior. In particular, it follows from the often very rapid convergence of the sum in Eq. (3) that details about the attractive fixed points may have almost no influence on physical observables, as long as the initial coupling lies sufficiently far from these fixed points.

In this Brief Report we shall consider some examples of $\mathrm{RG}$ flows that in certain regions depend very sensitively on an external parameter $p$. In particular we shall find several of the phenomena known from the study of complex dynamical systems: bifurcations of fixed points, chaotic regimes, and intermittency. The object of our investigation is to see to what extent such drastic effects on the RG flow have any bearing on physical observables.

The model we shall focus on is defined by "spins" $\operatorname{Tr} W_{j}$ with $W_{j} \in G$ (where $G$ is a compact Lie group) and $j$ indicates a site on a hypercubic lattice in $d$ dimensions. We take the trace in the fundamental representation of the group. The partition function is defined by

(C) 1991 The American Physical Society 
$Z=\int\left(d W_{j}\right) \exp \left[\frac{1}{2} J \sum_{\langle i, j\rangle}\left(\operatorname{Tr} W_{i} \operatorname{Tr} W_{j}^{\dagger}+\operatorname{Tr} W_{i}^{\dagger} \operatorname{Tr} W_{j}\right)\right]$,

where the integral is performed over the left and right invariant Haar measure of the group $G$ at all sites $j$. (The model describes the deconfinement phase transition of strongly coupled lattice gauge theories [3], but this application is of no relevance for what follows.) We shall restrict ourselves to the groups U(1) and SU(2). For U(1) the model is equivalent to the $d$-dimensional XY model.

Our framework shall be that of Kadanoff's variational lower-bound real-space renormalization group (LBRG) [4,5] (see, e.g., Ref. [6] for a well-written review). This method, which combines bond moving with an optimization of the free energy by means of a variational parameter $p$, is known to be extremely accurate for a host of discrete spin models [6]. Hierarchical lattices [7] can be constructed for which it becomes exact.

The LBRG restricts all interactions to lie within one $d$-dimensional hypercube. For a spin model of just a discrete symmetry group this automatically guarantees that the operator basis on which the LBRG is defined is finite. For a Lie group valued spin model such as the one of Eq. (4) this is not the case, and an operator truncation is necessary $[8,9]$. Although this further approximation can reduce the accuracy and reliability of the method $[9,10]$, it will not be our concern here. We shall simply use the model to illustrate explicitly the issues discussed above. In fact, we are almost completely certain that none of the nontrivial modifications of the RG flow we shall encounter below (even if they should have physical consequences) would reflect true behavior of the thermodynamic system described by Eq. (4). But the resulting $\mathrm{RG}$ equations satisfy all thermodynamic requirements, and they can therefore be used to perform reliable computations.

We start by considering the model corresponding to $G=\mathrm{SU}(2)$ in the case of $d=2$ dimensions. In a truncation to at most four spin interactions on the hypercube, we can write on this hypercube the most general $Z(2)$ even (see below) SU(2) invariant Hamiltonian which is symmetric under the exchange of any two spins as

$$
\begin{aligned}
h(W)= & K_{0}+K_{21} \sum_{i} W_{i}^{2}+K_{22} \sum_{\substack{i, j \\
i<j}} W_{i} W_{j}+K_{41} \sum_{i} W_{i}^{4} \\
& +K_{42} \sum_{i, j}^{\prime} W_{i}^{3} W_{j}+K_{43} \sum_{\substack{i, j \\
i<j}} W_{i}^{2} W_{j}^{2} \\
& +K_{44} \sum_{\substack{i, j, k \\
i, j<k}} W_{i}^{2} W_{j} W_{k} \\
& +K_{45} \sum_{\substack{i, j, k, l \\
i<j<k<l}} W_{i} W_{j} W_{k} W_{l} .
\end{aligned}
$$

Here we have made use of the fact that the irreducible representations of $\mathrm{SU}(2)$ are real, and we have defined a new variable $W_{i}^{\text {new }} \equiv \operatorname{Tr} W_{i}^{\text {old }}$. A prime on the summation symbol indicates that equal spins are not included. At a possible phase transition it is not $\mathrm{SU}(2)$, but only the discrete global $Z(2)$ invariance $W_{i} \rightarrow \pm W_{i}$ (all $i$ ) that gets spontaneously broken. The Hamiltonian $h(W)$ above contains only operators even under $Z(2)$. The LBRG is stable under the restriction to the symmetric subspace of Hamiltonians.

First define $\not_{0} \equiv \int\left(d Z_{i}\right) \mathcal{K}(Z, p)$ and

$$
\partial_{n_{1}} \cdots n_{\alpha} \equiv \partial_{0}^{-1} \int\left(d Z_{i}\right) Z_{i}^{n_{1}} \cdots Z_{\alpha}^{n_{\alpha}} \mathcal{K}(Z, p),
$$

where

$$
\mathcal{K}(Z, p)=\frac{\exp \left[4\left(h(Z)-K_{0}\right)\right]}{I_{0}\left(2 p \sum_{i} Z_{i}\right)-I_{2}\left(2 p \sum_{i} Z_{i}\right)}
$$

and $I_{n}(x)$ denotes the modified Bessel function of order $n$. The LBRG recursion relations then read [9]

$$
\begin{aligned}
& K_{0}^{\prime}=4 K_{0}+\ln \partial_{0}, \quad K_{42}^{\prime}=\frac{p^{4}}{2}\left(\partial_{31} / 3-\partial_{2} \partial_{11}\right), \\
& K_{21}^{\prime}=\frac{p^{2}}{2} \partial_{2}, \quad K_{43}^{\prime}=\frac{p^{4}}{4}\left(\partial_{22}-\partial_{2}^{2}-2 \partial_{11}^{2}\right), \\
& K_{22}^{\prime}=p^{2} \partial_{11}, \quad K_{44}^{\prime}=\frac{p^{4}}{2}\left(\partial_{211}-2 \partial_{11}^{2}-\partial_{2} \partial_{11}\right), \\
& K_{41}^{\prime}=\frac{p^{4}}{8}\left(\partial_{4} / 3-\partial_{2}^{2}\right), \quad K_{45}^{\prime}=p^{4}\left(\partial_{1111}-3 \partial_{11}^{2}\right) .
\end{aligned}
$$

If the method did not involve bond moving or operator truncation, all final results should be independent of the variational parameter $p$, which here enters only from the projection operator linking the initial and renormalized Hamiltonians. Instead of optimizing the free energy with respect to $p$, we shall simply treat $p$ as a free parameter with which we can "tune" the RG flow. For the present purpose it is most instructive to focus on $p$ values in the neighborhood of $p=0.39$. There are two attractive fixed points ( $A$ and $B$ ), and one critical fixed point $C$ in an interval $0.383 \ldots \leq p \leq 0.395 \ldots$. For $p \leq 0.383 \ldots$ we have just one fixed point $(A)$, while for $p \geq 0.395$. . we again have only one fixed point $(B)$. We illustrate this in the flow diagram of Fig. 1. The present approximate RG analysis thus leads to only one phase for both $p$ values chosen. But this single phase is evidently associated with two different attractive fixed points, which one can shift between by very tiny adjustments of the parameter $p$. How does this change in the stability of the fixed points reflect itself in the thermodynamics of the original model? We answer this question by computing the free energy $f$ from Eqs. (3) and (7) for $p=0.382$ and 0.396. Only for $K_{22}$ values around -0.36 do we find a (very small) difference, which is simply caused by a mild $p$ dependence of the remnant of the phase transition that exists for all $p$ values inbetween. In order to see this more clearly, we show in Fig. 2 the specific heat $\chi=K_{22}^{2} \partial^{2} f / \partial K_{22}^{2}$. The whole effect is at the $1-2 \%$ level, and is precisely as expected from the above remarks. It is not difficult to understand the cause of this insignificant change in $f(K)$ : Whether one flows indefinitely toward the fixed point $A$ or first flows toward $A$ and then, only after a very large number of iterations, bends off toward $B$, is of no relevance for the functional Eq. (3), on account of the 

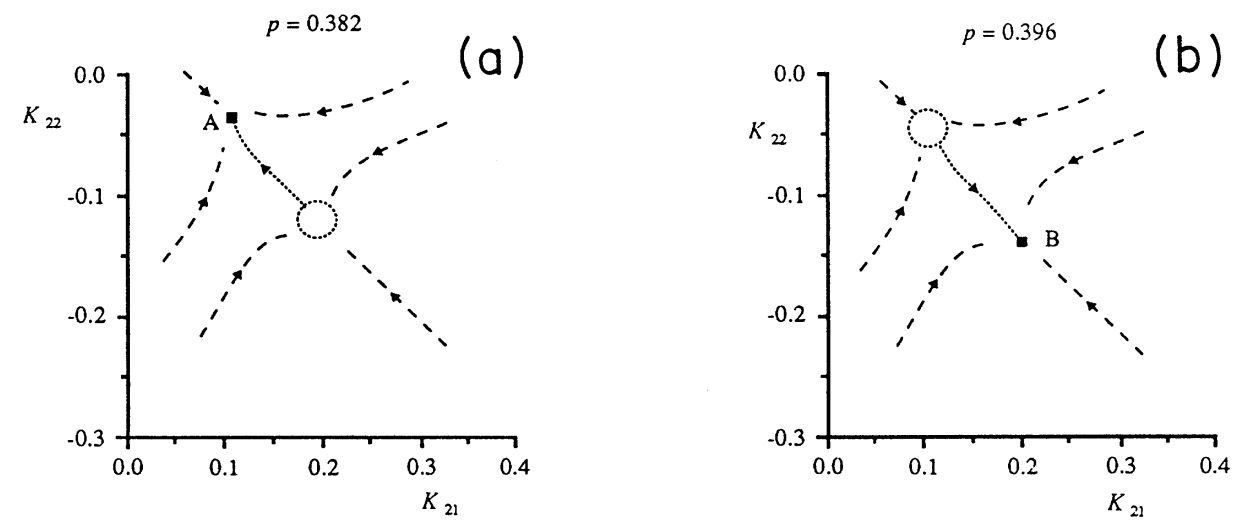

FIG. 1. The schematic flow diagram of the model (4) for $G=\mathrm{SU}(2)$, with $p=0.382$ and $p=0.396$ for (a) and (b), respectively.

rapid convergence in this region. In effect, what we have learned is that a shift between intermittent behavior around $A$ and true convergence toward $A$ is not of great import for the free energy.

Finally consider the model (4) for the group U(1) in $d=2$ dimensions. By again expanding on a basis of up to four spin interactions, one can in this case obtain a closed analytic expression for the recursion relations of the LBRG $[8,10]$ :

$$
\begin{aligned}
K_{0}^{\prime}= & 4 \ln \left[2 \pi I_{0}(2 p)\right]+4 K_{0}-u_{0}(p) \\
+ & 3\left\{1-\left[r_{2}(p)\right]^{2}\right\}\left\{\frac{1}{2}\left[8 K_{2}-u_{2}(p)\right]^{2}+16 K_{41}\right. \\
& \left.-u_{4}(p)\right\}
\end{aligned}
$$

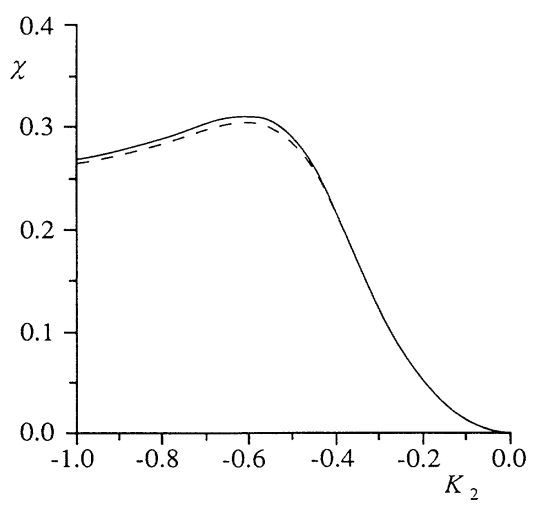

FIG. 2. The specific heat $\chi$ evaluated for the two different attractive fixed points in the $\mathrm{SU}(2)$ model for the same $p$ values as in Fig. 1. where the coupling constants are defined analogously to Eq. (5) [10],

$$
\begin{aligned}
& r_{n}(p)=I_{n}(2 p) / I_{0}(2 p), \\
& u_{0}(p)=\ln \left[I_{0}(4 p)\right], \\
& u_{2}(p)=p I_{1}(4 p) / 2 I_{0}(4 p),
\end{aligned}
$$

and

$$
u_{4}(p)=p^{2} I_{2}(4 p) / 8 I_{0}(4 p)-\left[u_{2}(p)\right]^{2} / 2 .
$$

For $p \geq 0.704$ we find the beginning of a bifurcation route to chaos in the system of recursion relations (8). In the chaotic regime the high- $T$ sink is replaced by a strange attractor [10]. Chaos begins at $p=0.820 . \ldots$, and ends at $p=0.842 \ldots$ as the result of a boundary crisis [11], where the critical hypersurface collides with the strange attractor.

Thus for $p \geq 0.704$. . we have a profound change in the RG flow as the high- $T$ stable fixed point becomes unstable through period-doubling sequences, and eventually becomes replaced by a completely chaotic attractor. How does this influence the original model (4)? In Table I we show how the free energy is obtained from Eq. (3) for three values of $p: p=0.60$ (only one attractive fixed point), $p=0.75$ (a period-two limit cycle), and $p=0.82$ (the chaotic regime). We have chosen the $K_{22}$ value at which the difference is the largest, which as expected occurs fairly close to the (former) attractive fixed point. It is obvious that neither the oscillatory behavior due to a stable limit cycle nor the chaotic behavior has any significant effect. (Recall that the free energy in any case is evaluated at different $P$ values, which always leads to some variation in $f$.) The cause of this remarkable stability of $f$ can again be found in the fact that all modifications of the RG flow occur in a region that is not immediately reachable from the nearest-neighbor interaction axis on which we evaluate the free energy. Of course one can encounter singular situations in which this region precisely coincides with the coupling-constant values at which one wishes to compute the thermodynamic functions. Only then may details of the flow close to the attractor become important for the free energy itself. This conclusion cannot be extended to $f^{(n)}$, the $n$th derivative 
TABLE I. Convergence of the iterative solution $f^{(n)}$ from Eq. (3) toward the full free energy $f$ of the U(1) model as a function of the number of iterations $n$. The three different situations I, II and III correspond to $p=0.60$ (one stable fixed point), $p=0.75$ (a period-two limit cycle), and $p=0.82$ (chaos), respectively.

\begin{tabular}{|c|c|c|c|c|c|c|c|c|c|}
\hline \multirow[b]{2}{*}{$n$} & \multicolumn{3}{|c|}{ I } & \multicolumn{3}{|c|}{ II } & \multicolumn{3}{|c|}{ III } \\
\hline & $K_{0} / 2^{\text {nd }}$ & $K_{2}$ & $f^{(n)}$ & $K_{0} / 2^{n d}$ & $K_{2}$ & $f^{(n)}$ & $K_{0} / 2^{n d}$ & $K_{2}$ & $f^{(n)}$ \\
\hline 1 & 1.7242 & -0.0225 & 0.2155 & 1.8851 & -0.0201 & 0.2356 & 1.9684 & -0.0171 & 0.2460 \\
\hline 2 & 2.1130 & -0.0392 & 0.2816 & 2.2892 & -0.0636 & 0.3072 & 2.3766 & -0.0789 & 0.3203 \\
\hline 3 & 2.2156 & -0.0326 & 2.2989 & 2.4069 & -0.0276 & 0.3260 & 2.5043 & -0.0118 & 0.3399 \\
\hline 4 & 2.2407 & -0.0365 & 0.3032 & 2.4321 & -0.0739 & 0.3307 & 2.5278 & -0.1145 & 0.3448 \\
\hline 5 & 2.2470 & -0.0347 & 0.3043 & 2.4396 & -0.0221 & 0.3319 & 2.5369 & 0.0475 & 0.3461 \\
\hline 6 & 2.2486 & -0.0357 & 0.3046 & 2.4411 & -0.0837 & 0.3322 & 2.5380 & -0.1337 & 0.3464 \\
\hline 7 & 2.2490 & -0.0352 & 0.3047 & 2.4416 & -0.0086 & 0.3323 & 2.5387 & 0.1018 & 0.3464 \\
\hline 8 & 2.2491 & -0.0355 & 0.3047 & 2.4417 & -0.0927 & 0.3323 & 2.5387 & -0.0763 & 0.3465 \\
\hline 9 & 2.2491 & -0.0354 & 0.3047 & 2.4418 & 0.0083 & 0.3323 & 2.5388 & -0.0228 & 0.3465 \\
\hline 10 & 2.2491 & -0.0354 & 0.3047 & 2.4418 & -0.0968 & 0.3323 & 2.5388 & -0.1104 & 0.3465 \\
\hline 11 & 2.2491 & -0.0354 & 0.3047 & 2.4418 & 0.0186 & 0.3323 & 2.5388 & 0.0327 & 0.3465 \\
\hline 12 & 2.2491 & -0.0354 & 0.3047 & 2.4418 & -0.0954 & 0.3323 & 2.5388 & -0.1424 & 0.3465 \\
\hline
\end{tabular}

of $f$ which eventually, for sufficiently large $n$, may reveal details of the nature of the attractor.

Chaotic renormalization-group trajectories have been observed earlier for related (but one-dimensional) realspace renormalization maps $[12,13]$. They have commonly been viewed as signalling the existence of a spin-glass phase [12], but an interpretation in terms of incommensurate structures has also been suggested [13]. A study of some of the physical effects of birfurcations and chaos around critical fixed points (again for maps with just one coupling constant) has been presented earlier in Ref. [14]. A general and systematic analysis of corresponding phenomena in the case of higher-dimensional renormalization-group transformations will be reported elsewhere [15].
[1] Th. Niemeijer and J. M. J. van Leeuwen, in Phase Transitions and Critical Phenomena, edited by C. Domb and M. S. Green (Academic, New York, 1976), Vol. VI.

[2] F. J. Wegner, J. Phys. C 7, 2098 (1974).

[3] F. Green and F. Karsch, Nucl. Phys. B 238, 297 (1984).

[4] L. P. Kadanoff, Phys. Rev. Lett. 34, 1005 (1975).

[5] L. P. Kadanoff, A. Houghton, and M. C. Yalabik, J. Stat. Phys. 14, 171 (1976).

[6] T. W. Burkhardt, in Real Space Renormalization, edited by T. W. Burkhardt and J. M. J. Van Leeuwen (Springer, Berlin, 1982).

[7] A. N. Berker and S. Ostlund, J. Phys. C 12, 4961 (1979); M. Kaufman and R. B. Griffiths, Phys. Rev. B 24, 496 (1981); J. Phys. A 15, L239 (1982).

[8] M. Droz and A. Malaspinas, J. Phys. C 11, 2729 (1978).
[9] G. Thorleifsson and P. H. Damgaard, J. Phys. A 23, 5863 (1990).

[10] G. Thorleifsson and P. H. Damgaard, Physica A (to be published).

[11] C. Grebogi, E. Ott, and J. A. Yorke, Phys. Rev. Lett. 48, 1507 (1982).

[12] S. McKay, A. N. Berker, and S. Kirkpatrick, Phys. Rev. Lett. 48, 767 (1982); A. N. Berker and S. McKay, J. Stat. Phys. 36, 787 (1984).

[13] N. M. Svrakic, J. Kertesz, and W. Selke, J. Phys. A 15, L427 (1982).

[14] B. Derrida, J.-P. Eckmann, and A. Erzan, J. Phys. A 16, 893 (1983).

[15] P. H. Damgaard (unpublished). 